\title{
Intelligent Monitoring System for Plant Growth Shelf Based on STM32
}

\author{
Kun Liu ${ }^{1,2+}$, Shigang Cui ${ }^{1,2}$, Jianhua Qin ${ }^{1,2}$, Xingli Wu ${ }^{1,2}$ and Lin $\mathrm{He}^{1,2}$ \\ ${ }^{1}$ Tianjin University of Technology and Education \\ ${ }^{2}$ Tianjin Key Laboratory of Information Sensing \& Intelligent Control
}

\begin{abstract}
An intelligent monitoring system for plant growth shelf was designed on embedded technology, which took the STM32 microprocessor as its central-controller. A touch screen was used to display the realtime temperature, humidity and $\mathrm{CO}_{2}$ concentration information. Multi-sensor data fusion was used to improve the accuracy of the sensor, and controlled the bright intensity of white LED lamp group by pulse width modulation (PWM) signal. The testing results showed that this intelligent monitoring system operated stable and reliable, the interface was friendly, and could satisfy the need for control.
\end{abstract}

Keywords: plant growth shelf; STM32; environmental factor; intelligent control.

\section{Introduction}

Plant factory is an efficient production method which can use intelligent automatic control technology to control the internal temperature, humidity, light, $\mathrm{CO}_{2}$ concentration, nutrient solution and other environmental factors according to the demand of agricultural crops growth environment[1]. It achieves the continuous production of annual crops, and has the characteristics of saving resources, safety and no pollution[2].

The plant factory is designed with closed space, and the internal temperature and humidity, $\mathrm{CO}_{2}$ concentration and other environmental factors are controlled by central control system. As the basic unit of production in the plant factory, the plant growth shelf is the planting area of the plant factory. Due to the large space inside the plant factory, environment in different spatial positions have certain differences, plant growth shelf in different position of plant factory can be used to monitor environmental factors, it plays an important role in environmental monitoring. The key to the development of plant growth shelf is to monitor the environmental factors such as temperature and humidity and $\mathrm{CO}_{2}$ concentration, and to design an accurate LED brightness control system. In view of this situation, this paper presents an intelligent monitoring system for plant growth shelf based on STM32. In this system, the micro-controller center STM32 is used, accurate measurement and real-time display of temperature, humidity and $\mathrm{CO}_{2}$ concentration, and the ability to accurately adjust the bright intensity of LED lamp panel, creating crop growth and development of the best light condition, the system is easy to operate monitoring, high precision and low cost.

\section{Overall design scheme}

Monitoring system is mainly composed of processor module, sensor module, LED control module, display screen and power supply module. The processor module is the core of the control part of the plant growth shelf, completes the system information processing and external control, and displays environmental data on the LCD screen; The sensor module is used to collect the environmental data inside the plant growth shelf; The LED control module controls the bright intensity of the LED lamp board by the set parameters;

\footnotetext{
+ Corresponding author. Tel.: 18678732556.

E-mail address: china68kun@163.com.
} 
The display screen is data output devices, it can display the environment data and bright intensity. The power supply module provides power for the whole system. Structural diagram of control system is as shown in Fig.1.

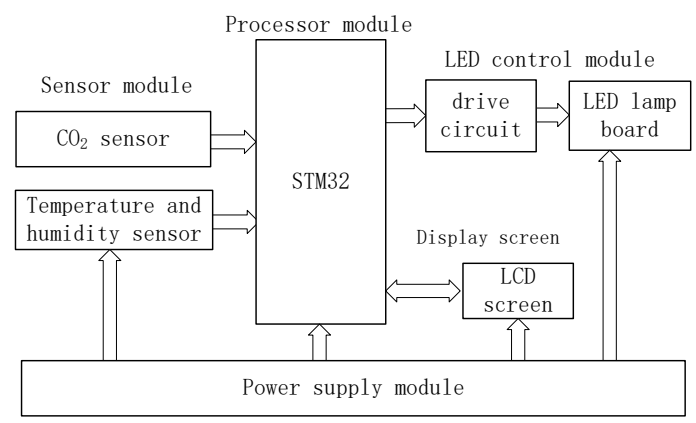

Fig.1: Structural diagram of control system

\section{System hardware design}

\subsection{Processor module}

The processor module takes the STM32F103VET6 microprocessor as its core controller, compared with similar products, the STM32 is a micro controller based on high performance and low power consumption, and it works at $72 \mathrm{MHz}$, with $512 \mathrm{~KB}$ FLASH, $64 \mathrm{~K}$ SRAM, and a Ethernet interface. What' $\mathrm{s}$ more, it also includes two RS232 ports. The system in our paper use the function library released by ST company, which reduce the development cycle and meet the design requirements of the system [3].

\subsection{Sensor module}

The sensor module mainly includes a temperature and humidity sensor and a $\mathrm{CO}_{2}$ sensor. Temperature and humidity sensor use AM2321B, which is a digital signal containing the calibration of the temperature and humidity sensor, with fast response, strong anti-interference ability, high reliability. AM2321B sensor use the $\mathrm{I}^{2} \mathrm{C}$ interface to connection with the STM32, and complete the corresponding monitoring work. $\mathrm{CO}_{2}$ sensor use MG811, which can complete the $0-10000 \mathrm{ppm}$ concentration measurement of $\mathrm{CO}_{2}$, the sensor corresponds to the analog output of $0-4 \mathrm{~V}$. What's more, the sensor has high sensitivity and good selectivity.

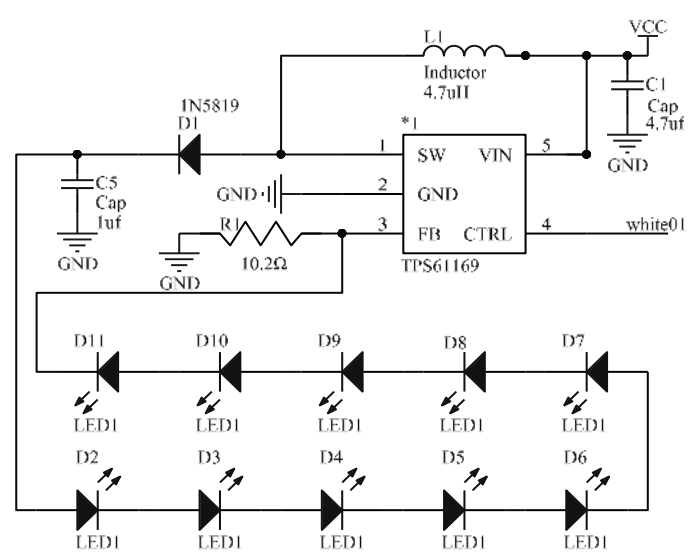

Fig.2: LED driver circuit schematic

\subsection{LED control module}

LED control module adopts multi group constant current drive circuit, using PWM control technology to realize the bright intensity control of LED lamp. The LED control module includes a LED lamp panel and an external drive circuit. The LED lamp is controlled by PWM signal and the drive circuit is controlled by TPS61169 module. LED driver circuit schematic is as shown in Fig. 2. LED light board contains a number of LED light group, with a rated power of $1 \mathrm{~W}$ white LED, through series connection. The bright intensity lamp group maximum duty cycle of PWM is 1 when the rated power output of LED. The PWM duty cycle and output current into a linear relationship, though setting the PWM signals with different duty cycle, the output 
current of the LED driver chip is changed, and realize the PWM duty cycle control the bright intensity of LED lamp panel.

\subsection{Display screen}

The display screen uses DGUS LCD screen as the display terminal, the screen resolution is $800 * 480$, the normal working voltage is $12 \mathrm{~V}$. The LCD has the characteristics of strong anti-interference and good electromagnetic compatibility. It uses the serial communication to connection with the STM32, real-time display of the temperature and humidity of the environment and $\mathrm{CO}_{2}$ concentration, while the bright intensity of LED can be set through the interface buttons.

\subsection{Power supply module}

Power supply module is an important part of the whole system, the system uses $24 \mathrm{~V}$ and $12 \mathrm{~V}$ output power switch for the LED light board and display power supply, $24 \mathrm{~V}$ is converted into $5 \mathrm{~V}$ though the transformer chip LM2596s, and $5 \mathrm{~V}$ is converted into $3 \mathrm{~V}$ though the LM1117, both of them achieve the power supply of the temperature and humidity sensor and STM32 micro controller.

\section{System software design}

\subsection{Data fusion algorithm}

A temperature and humidity sensor and a carbon dioxide sensor are arranged on each layer of the plant growth shelf[4]. However, the sensor is easy to be interfered by external factors and is influenced by its accuracy, so the test result can't reflect the real state. The traditional single sensor monitoring technology is difficult to accurately collect the data of temperature and humidity, and the multi-sensor cooperative work must be adopted to complete the monitoring task. Statistical studies show that the sample observations from the single sensor system have the characteristics of normal distribution, which can be regarded as equal precision. In order to obtain relatively accurate data, the fusion algorithm based on batch estimation is used to process the measured values of each layer[5]. For example, a series of temperature data are obtained by 8 measurements in the plant growth frame: $T_{1}, T_{2}, T_{3}, T_{4}, T_{5}, T_{6}, T_{7}, T_{8}$, the data was divided into 2 groups, respectively including $T_{1}, T_{3}, T_{5}, T_{7}$ and $T_{2}, T_{4}, T_{6}, T_{8}$, the average values $\bar{T}_{1}, \bar{T}_{2}$ and standard deviation $\sigma_{1}, \sigma_{2}$ of two groups were:

$$
\begin{aligned}
& \bar{T}_{1}=\frac{1}{4} \sum_{i=1}^{4} T_{2 i-1} \\
& \bar{T}_{2}=\frac{1}{4} \sum_{i=1}^{4} T_{2 i} \\
& \sigma_{1}=\sqrt{\frac{1}{4-1} \sum_{i=1}^{4}\left(T_{2 i-1}-\bar{T}_{1}\right)^{2}} \\
& \sigma_{2}=\sqrt{\frac{1}{4-1} \sum_{i=1}^{4}\left(T_{2 i}-\bar{T}_{2}\right)^{2}}
\end{aligned}
$$

According to the research of literature[5], the batch estimation theory in the statistics can be used to solve the fusion value $T^{+}$of the data according to formulas (1), (2), (3) and (4):

$$
\mathrm{T}^{+}=\frac{\sigma_{1}^{2} \bar{T}_{2}+\sigma_{2}^{2} \bar{T}_{1}}{\sigma_{1}^{2}+\sigma_{2}^{2}}
$$

\subsection{Main design program}

The traditional monitoring system mostly adopts the front and back mode, this paper uses the embedded operating system uC/OS-II and the software design method is used to ensure the reliability and stability of the system. The system contains five tasks such main task of the system, data acquisition, data process, communications and touch screen. The main task is to set up by calling the function; data acquisition is to mainly used to collect the temperature and humidity data regularly; Data process is to collect data for data fusion; Communication is to achieve LCD screen and STM32 communication connection; Touch screen is to display temperature, humidity and $\mathrm{CO}_{2}$ concentration, and adjust the bright intensity of LED lamp panel. 


\section{Test analysis}

\subsection{Monitoring system test}

The display interface of the monitoring system is shown in Fig.3 The main text on the left side of the LCD screen shows the 4 layer of the current bright intensity, the right side of the 3 small text box in realtime display of temperature, humidity and $\mathrm{CO}_{2}$ concentration information. Users click the set button to enter the LED control system, as shown in Fig.4. The upper part shows the current value, the lower can set the bright intensity of LED.

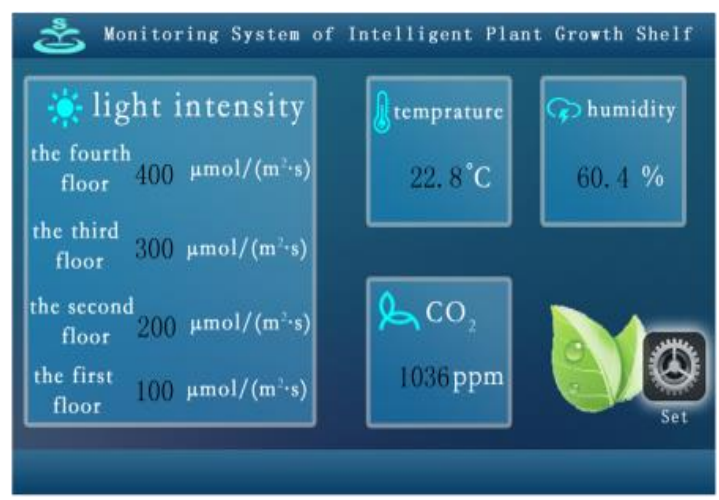

Fig.3: Screen monitoring system interface

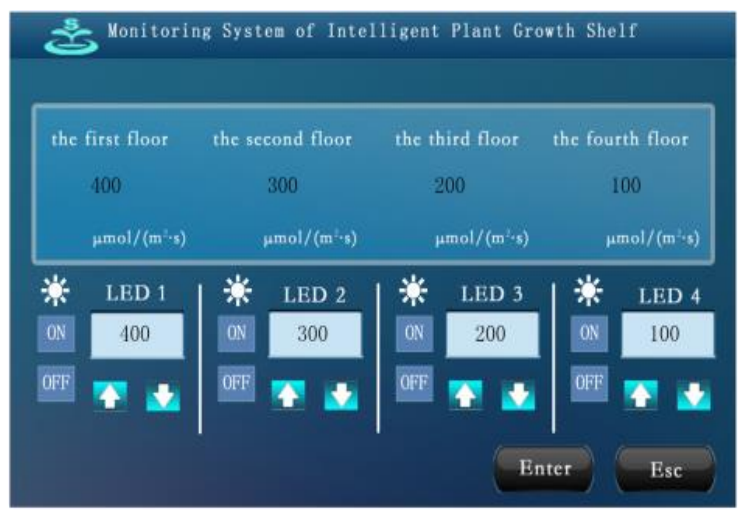

Fig.4: Screen control system interface

\subsection{Data fusion algorithm test}

The area of the plant factory was $15 \mathrm{~m}^{2}$, and the environmental parameters of temperature, humidity, $\mathrm{CO}_{2}$ concentration were respective $22^{\circ} \mathrm{C}, 60 \%, 1000 \mathrm{ppm}$. The monitoring data of temperature, humidity and $\mathrm{CO}_{2}$ concentration of plant growth shelf based on multi-sensor data fusion algorithm as shown in Table 1.

Table 1: Monitoring data

\begin{tabular}{cccc}
\hline & \multicolumn{3}{c}{ Fusion value } \\
\cline { 2 - 4 } Time/h & $\begin{array}{c}\text { Temperature }\left({ }^{\circ} \mathrm{C}\right. \\
\text { Humidity } \\
(\%)\end{array}$ & $\begin{array}{c}\mathrm{CO}_{2} \\
(\mathrm{ppm})\end{array}$ \\
\hline 0 & 23.2 & 60.4 & 1025 \\
2 & 23 & 60.7 & 1046 \\
4 & 22.8 & 61.5 & 1036 \\
6 & 22.5 & 60.5 & 1003 \\
8 & 23.1 & 60.1 & 984 \\
10 & 23.3 & 59.2 & 963 \\
12 & 23.4 & 59.7 & 1056 \\
14 & 23.5 & 60 & 1067 \\
16 & 23.2 & 60.2 & 1016 \\
18 & 22.8 & 60.5 & 1005 \\
20 & 23.3 & 60.4 & 981 \\
22 & 23.2 & 60.2 & 1025 \\
\hline
\end{tabular}

As can be seen from table.1, the system can successfully monitor the temperature, humidity and $\mathrm{CO}_{2}$ concentration data in the region. Data show that the temperature error of $24 \mathrm{~h}$ within the range of $\pm 0.5^{\circ} \mathrm{C}$, the humidity error within $2.5 \%$ range, carbon dioxide concentration error is less than $5 \%$, the monitoring results reach the design goals and meet the needs of actual projects. 


\section{Conclusion}

In order to meet the needs of plant growth shelf, an intelligent monitoring system based on STM32 was designed. In this system, accurate measurement and real-time display of temperature, humidity and $\mathrm{CO}_{2}$ concentration, multi-sensor data fusion was used to improve the accuracy of the sensor. The ability to accurately adjust the bright intensity of LED lamp panel by pulse width modulation (PWM) signal. The system has the advantages of reliable system performance, friendly display interface, accurate control and high practicability.

\section{Acknowledgements}

This work was supported by 863 Projects (Project No.2015AA033303), the Science, Technology Development Fund Project of TUTE(Project No.YC17-25) and the Foundation of Tianjin University of Technology and Education (Project No.KYQD12012)

\section{References}

[1] Yang Qichang, Wei Lingling, Liu Wenke et al. Plant factory system and practice [M]. Chemical Industry Press, 2012.

[2] He Dongxian, Zhu Benhai, Yang Po et al. Design and environment control of closed plant factorieswith artificial 1 ighting[J]. Transactions of the CSAE, 2007, 23(3):151-157.

[3] Ai Haibo, Wei Jinhong, Qiu Quan, et al. Design of miniature plant factory temperature and humidity monitoring system of based on STM32[J]. Journal of Agricultural Mechanization Research, 2014(5): 141-144.

[4] Zhu Mingxiang, Xu Xiaobin, Chen Zhaozhang. Design of constant temperature box measurement and control system based on multi-sensor information fusion[J]. Transducer and Microsystem Technologies, 2010, (03): 62$64+67$.

[5] Fei Yetai. Error theory and data processing[M]. Hefei: Press of University of Science and Technology of China, 1999: 78-92.

[6] Li Hengcan, Li Quancai. Application of Data Fusion in Monitoring Environment[J]. Chinese Agricultural Mechanization, 2011,(04):110-113+118. 